\title{
Rationale, Development, and Design of the Altering Intake, Managing Symptoms (AIMS) Dietary Intervention for Bowel Dysfunction in Rectal Cancer Survivors
}

\author{
Virginia Sun, PhD, RN ${ }^{1}$, Tracy E. Crane, PhD, RDN ${ }^{2,3}$, Samantha D. Slack, BS ${ }^{3,4}$, \\ Angela Yung, BS, $\mathrm{RDN}^{3}$, Sarah Wright, $\mathrm{BS}^{3,4}$, Stephen Sentovich, $\mathrm{MD}^{5}$, Kurt Melstrom, $\mathrm{MD}^{5}$, \\ Marwan Fakih, $\mathrm{MD}^{6}$, Robert S. Krouse, $\mathrm{MD}^{7}$, Cynthia A. Thomson, PhD, RDN ${ }^{3,4}$
}

\author{
Author Affiliations: \\ ${ }^{1}$ Department of Population Sciences, City of Hope, Duarte, CA \\ ${ }^{2}$ College of Nursing, University of Arizona, Tucson, AZ \\ ${ }^{3}$ University of Arizona Cancer Center, Tucson, AZ \\ ${ }^{4} \mathrm{Mel}$ and Enid Zuckerman College of Public Health, University of Arizona, Tucson, AZ \\ ${ }^{5}$ Division of Surgical Oncology, Department of Surgery, City of Hope, Duarte, CA \\ ${ }^{6}$ Department of Medical Oncology and Therapeutics Research, City of Hope, Duarte, CA \\ ${ }^{7}$ Surgical Services, Corporal Michael J. Crescenz Veterans Affairs Medical Center and Perelman \\ School of Medicine, University of Pennsylvania, Philadelphia, PA
}

Correspondence: Virginia Sun, PhD, RN, Assistant Professor, Division of Nursing Research and Education, Department of Population Sciences, City of Hope, 1500 East Duarte Road, Duarte, CA 91010, USA; Tel: 626/218-3122; fax: 626/218-8941; email: vsun@coh.org 


\begin{abstract}
Purpose: Bowel dysfunction is a common, persistent long-term effect of treatment for rectal cancer survivors. Survivors often use dietary modifications to maintain bowel control. There are few evidence-based interventions to guide survivors on appropriate diet modifications for bowel symptom management. The purpose of this paper is to describe the development and design of the Altering Intake, Managing Symptoms (AIMS) intervention to support bowel dysfunction management in rectal cancer survivors.
\end{abstract}

Methods: The AIMS intervention is a ten-session, telephone-based diet behavior change intervention delivered by trained health coaches. It uses dietary recall, participant-completed food and symptom diaries, and health coaching guided by motivational interviewing to promote bowel symptom management and improved diet quality. Based on the Chronic Care SelfManagement Model (CCM), the AIMS Intervention is designed to improve self-efficacy and self-management of bowel symptoms by coaching survivors to appropriately modify their diets through goal setting, self-monitoring, and problem-solving. The intervention targets survivors with stage I-III rectosigmoid colon/rectum cancer who are 6 months post-treatment, 21 years and older, and English-speaking.

Conclusions: The design and development process described in this paper provides an overview and underscores the potential of the AIMS intervention to positively impact the quality of longterm survivorship for rectal cancer survivors. An ongoing pilot study will inform the design and development of future multi-site Phase II and III randomized trials.

Key Words: rectal cancer; survivorship; diet; bowel dysfunction; telephone behavior counseling; symptom management 


\section{Introduction}

There are more than 1.4 million Americans living with a history of colorectal cancer ${ }^{1}$; an additional 135,400 cases will be diagnosed in $2017 .^{2}$ Compared to colon cancer, incidence of rectal cancer is lower, with nearly 39,000 estimated new cases in 2017. For survivors with rectal cancer, the standard treatment often involves multi-modality regimens that include neoadjuvant chemoradiation, surgery, followed by adjuvant chemotherapy. Bowel resection is a cornerstone of curative therapy for rectal cancer.

Advances in treatment and surgical techniques have resulted in longer survival and cure rates in rectal cancer. However, survival comes at a price for many. Survivors often experience multiple, persistent long-term effects of treatment. Functional deficits, primarily in relation to bowel dysfunction, result in dramatic deteriorations in health-related quality of life (HRQOL) for rectal cancer survivors. ${ }^{3-5}$ Bowel dysfunction is one of the most common and debilitating longterm effects of surgery and multimodality treatment. About $27 \%$ to $56 \%$ of rectal cancer survivors report living with persistent bowel dysfunction symptoms more than 1-2 years after their surgery and treatment. ${ }^{4,6-9}$ A constellation of unpleasant symptoms are associated with bowel dysfunction; these include fecal incontinence, urgency, frequency, sense of incomplete fecal evacuation, oscillations from diarrhea to constipation, excessive gas, bloating, colitis, and abdominal cramping. ${ }^{10}$ The etiology of bowel dysfunction is multifactorial; surgically, changes in the gastrointestinal (GI) tract anatomy and gut microbiome are associated with bowel symptoms. ${ }^{9}$ Radiation and chemotherapy often exacerbate these symptoms. ${ }^{8,10}$

A common self-care strategy used by rectal cancer survivors to manage bowel symptoms is diet modification. Our previous research suggests that successfully managing bowel symptoms results in improved HRQOL. ${ }^{4}$ However, in the absence of structured guidance, the choice of diet 
modifications was often based on a trial-and-error approach that yielded inconsistent results. In addition, survivors reported tremendous variations in the diet modification strategies applied. Fruits and vegetables were the most commonly cited foods for diet modifications; however, these food items were reported to be both helpful and troublesome in regard to bowel symptoms. ${ }^{11}$ Often, survivors eliminate troublesome foods that exacerbate bowel symptoms, and avoid foods including select vegetables and fruits because of an association with bowel problems. ${ }^{11}$ Importantly, bowel symptoms and diet behaviors are modifiable. Many cancer survivors are motivated to change their behaviors to reduce recurrence and improve overall survival. ${ }^{12}$ The benefits of a healthy diet extends beyond improving oncologic outcomes in rectal cancer ${ }^{13-15}$; studies have shown that it may also lead to improvements in overall well-being, and reductions in co-morbid conditions such as cardiovascular disease and diabetes. ${ }^{16,17}$

There is a lack of evidence-based interventions to manage bowel dysfunction in rectal cancer survivors. The American Cancer Society (ACS) Colorectal Cancer Survivorship Guidelines rates bowel dysfunction management in the category 3 evidence level (case reports and case studies only). ${ }^{18}$ The purpose of this paper is to describe the rationale, development, and design of a pilot study to determine the feasibility and acceptability of the Altering Intake, Managing Symptoms (AIMS) dietary intervention for bowel dysfunction in rectal cancer survivors.

\section{Materials and Methods}

\section{Study Design}

The study is a single group, mixed-methods, pre-and post-intervention pilot study of the AIMS intervention in 10 rectal cancer survivors. The sample size was determined based on a balance between a sufficient sample needed to obtain feasibility data as well as the need to 
realistically complete the study in a 12 month timeframe. Based on the ORBIT model for developing behavioral treatments for chronic disease, a small sample using accessible subjects is acceptable for Phase IIa proof-of-concept studies. ${ }^{19}$ The primary objective of the study is to determine the feasibility of the intervention as measured by the percentage of rectal cancer survivors who agree to participate and who are able to complete $>80 \%$ of the intervention. The $80 \%$ intervention session completion metric is based on our previous experience with behavioral interventions. ${ }^{20}$ Our goal is to determine whether participants can complete at least 8 out of the 10 planned telephone sessions. The secondary objectives are 1) to determine the acceptability of the intervention through semi-structured exit interviews with participants; and 2) to examine survivor outcome patterns suggesting the potential efficacy of the intervention on bowel function, diet quality and HRQOL.

\section{Study Population}

\section{Eligibility Criteria}

The study aims to enroll survivors with a history of stage I-III cancers of the rectosigmoid colon or rectum. Other inclusion criteria include survivors who are 1) 6 months post-treatment completion, 2) have either a permanent ostomy (surgically-created opening in the body for the discharge of fecal wastes) or anastomosis, and 3) over 21 years of age. Participants who are non-English speaking will be excluded from the study.

\section{Recruitment Strategies}

This is a multi-site study involving two NCI-designated comprehensive cancer centers. The protocol was approved by the Institutional Review Boards (IRB) of both participating sites. Intervention delivery is centrally-administered at the site in Arizona. Participant recruitment is conducted at the site located in Southern California. The enrollment site serves a large catchment 
area with diverse patient populations by ethnicity and socioeconomic status. Participants are recruited through referrals from medical oncologists, surgical oncologists, and other clinical providers. Informed consent is obtained prior to study participation in accordance with national Human Subjects’ protection guidance. Baseline assessment surveys are administered following informed consent.

\section{Study Intervention}

\section{Conceptual Framework}

The AIMS Intervention is based on the Chronic Care Self-Management Model (CCM). ${ }^{21-}$ ${ }^{23}$ The CCM transforms a reactive health system into one that improves survivor outcomes through proactive planning and diet modification skills building. ${ }^{24}$ It focuses on skills building to empower and engage individuals in their own care. The model was originally developed as a response to the management of traditional chronic conditions such as diabetes and heart disease. ${ }^{23,25}$ It defines chronic conditions as any condition that requires ongoing adjustments by the affected person and interactions with the health care system. ${ }^{23}$ Cancer can be conceptualized as a chronic condition, as living with a cancer diagnosis requires constant adjustments and interactions with care providers along the continuum of care. ${ }^{26}$ The aim of the CCM is to transform a reactive health care system to a proactive one that is focused on keeping the patient as healthy as possible. ${ }^{27}$

In addition to the CCM, the AIMS intervention also builds upon classic behavioral change theory and approaches (see Figure 1). The AIMS intervention integrates key components of social cognitive theory and social determination theory to promote appropriate diet behavior change for symptom management. In Social Cognitive Theory, eating behavior is a function of interactive modeling and observed learning. Self-efficacy is achieved when knowledge gained is 
reinforced. As a cancer survivor practices the modeled behavior, self-efficacy is increased, and appropriate diet modifications and behavior changes are achieved. ${ }^{28}$

These theories and principles are predicated on several factors. First, an individual must make multiple, repeated choices on an hourly/daily basis to guide eating behaviors. Second, the decisions to follow a healthy diet, whether for symptom management or survivorship reasons, is both biological and behavioral. ${ }^{28}$ From a biological perspective, the influence of endocrine, metabolic, and hormonal pathways on eating behavior is heterogeneous; therefore, behavioral change interventions may have highly variable responses. ${ }^{28}$ This suggests that dietary interventions should be personalized to biological and physiologic profiles of each survivor. Rectal cancer survivors come with varying degrees of bowel symptomatology, treatment history, and other clinical factors (i.e. co-morbid conditions), which can all contribute to their diet behavior change experience. From a behavioral perspective, food preferences, food aversions, knowledge related to healthy diet, as well as sociocultural factors can all influence diet modifications and food intake. For rectal cancer survivors, experience with other symptoms during treatment, such as anticipatory nausea and vomiting, may also influence food choices and modifications.

Several well-established behavioral approaches are integrated in the AIMS intervention to promote appropriate diet modifications. Participants are coached to identify and set goals in relation to their diet modifications. Goal setting provides clarity for participants and coaches in terms of structure and expected outcomes. ${ }^{28}$ Both long-term and short-term goals are used for the intervention. Goal setting is combined with self-monitoring using a food and symptom diary to promote self-efficacy in diet behavior change. Self-monitoring provides survivors and their coach with a source of repeated awareness and cues for bowel control and healthy eating that can 
used to inform prospective goals. ${ }^{28}$ Identification of problems and challenges related to diet behavior change is addressed throughout the AIMS intervention. The health coaches work with the participants to identify potential challenges and problems; the key here is that survivors are the ones to identify the problems as well as potential solutions rather than the coaches. This patient-centered approach for problem-solving addresses the individual's previous approaches to overcome the identified challenges. ${ }^{28}$ Ultimately the survivor and coach define new realistic and adoptable strategies to achieve diet behavior change and symptom control. ${ }^{28}$

\section{Intervention Content and Procedures}

The AIMS intervention provides one-on-one telephone-based, health coaching to support diet modifications for rectal cancer survivors for bowel health and healthy diet through survivorship. As previously described, the coaching incorporates cognitive behavioral therapy that is operationalized through motivational interviewing techniques to deliver behavior-based, dietary interventions for symptom management. Motivational interviewing, based on Social Cognitive Theory, is an effective strategy employed in behavior change interventions. It is a collaborative, person-centered model to elicit and strengthen motivation for change. ${ }^{29}$ The intervention focuses on diet/symptom knowledge building for rectal cancer survivors, and supports behavior change with structured feedback, re-assessments, and goal setting.

The AIMS intervention is delivered within 4 months through 10 telephone sessions that last 30-40 minutes each (Table 1). Session 1 is delivered by a trained research assistant, who provides an overall introduction of the study, use of the food and symptom diary, and completes a 24-hour diet recall. The recall provides an assessment of the participant's overall diet quality. A fully developed intervention workbook with support reference materials and intervention content is provided to each participant after session 1. 


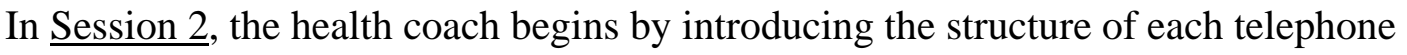
session. The workbook is introduced and reviewed with participants. Using the food and symptom diary, participants are coached to accurately document their food intake and note any symptoms associated with the foods. SMART (Specific, Measurable, Attainable, Relevant, Timely) goals for diet behavior change in relation to symptom management are identified. For example, a SMART goal may include eliminating a specific identified food for the next 7 days until the next telephone session, and to continue recording food intake and associated symptoms.

Sessions 3-6 are weekly calls. During these calls, the health coach uses the participant's bowel symptom experience to guide intervention delivery. Participants review their food/symptom diary and SMART goals with the health coach. The diary information is used to steer discussion on the elimination/substitution process of possible troublesome foods that cause bowel symptoms. Presence of symptom involves a careful look at the food/symptom diary, as well as problem-solving to integrate other symptom management strategies beyond diet modifications (e.g. sitz baths, fiber supplements).

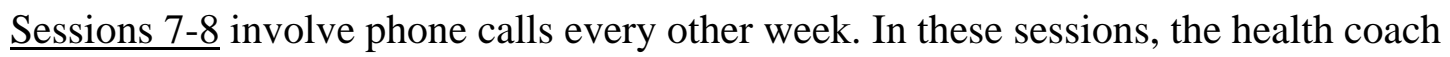
focuses on reintroducing, on a schedule, identified foods. The elimination/re-introduction diet process helps survivors identify the main food "culprits" that cause bowel symptoms, but also provides the skills with re-introducing other foods that are tolerable and beneficial. Participants are also coached to use problem-solving skills for overcoming diet behavior change challenges. This includes: 1) identification of perceived barriers to behavior change, 2) prior plans or strategies to overcome these barriers, and 3) identification of new strategies that are adoptable as part of diet modification. Evidence-based diet recommendations for cancer survivorship are also introduced. ${ }^{30,31}$ 


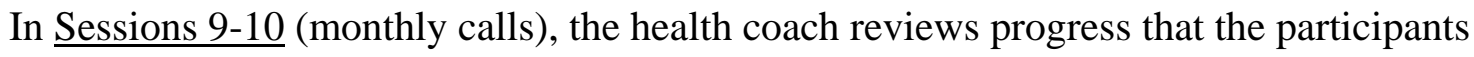
made and the skills they have gained to re-inforce self-efficacy for long-term application of the intervention. The health coach also revisits the intervention workbook to look at broader health topics (e.g. hydration, supplements) and review using SMART goals for appropriate diet change. A 24-hour diet recall is completed at 2 months post-intervention completion to assess diet quality.

\section{Treatment Fidelity, Intervention Adherence, and Quality/Process Evaluation}

Several approaches are included to ensure intervention rigor and validity. Standard operating procedures (SOP) outline implementation goals and strategies. The intervention is delivered by trained health coaches of the Behavioral Measurement and Interventions Shared Resource (BMISR) at the Arizona Cancer Center, under the direction of registered dietitian nutritionists. The health coaches have extensive training in motivational interviewing and phone communication experience in large scale, multi-site behavior change interventions. The centralized intervention administration model supports the feasibility of conducting future multisite Phase II and Phase III trials of the intervention. Health coaches were trained by the PI and co-investigators in May 2017 on study protocols and rectal cancer specific knowledge.

For intervention fidelity monitoring, a RedCap database was created and serves as a key communication portal between the health coaches and investigative team. Coaching call notes are entered into the system for each participant. All coaching sessions are audio-recorded, and the recordings are saved in the database for the PI and co-investigators to review. Based on the reviews, reinforcement and re-training are discussed as needed during weekly investigative team conference calls. Health coaches also complete, at the end of each telephone session, a coach debriefing form. This form is designed to assess level of participant intervention engagement. It 
includes 7 scored items and 2 open-ended questions. The form documents session length, and assesses, on a scale of 1-5 (1=very poor, 5=very good), in the coach's opinion, how well participants responded to the intervention sessions.

Self-reports through 24-hour dietary recalls and food/symptom diaries are used for intervention adherence monitoring. ${ }^{32}$ The dietary recalls are completed by trained coaches before the intervention and at 4 months post-intervention. Retention strategies were selected based on previous experience and published recommendations. ${ }^{33}$ First, study staff responsible for recruitment and study implementation are trained to provide clear information on study participation during the informed consent process, with particular emphasis on the potential benefits of participation. Second, scheduling control of the 10 telephone sessions is given to the participants. The length of each telephone session is also driven by the participant's specific needs. We also provided flexibility for data collection, utilizing multiple approaches (i.e. phone calls, mailings) to obtain outcomes data from participants.

\section{Outcome Measures}

The surveys used to obtain outcomes were selected to be patient-centered, validated, and relevant to the proposed conceptual framework and theories. The estimated time to complete the surveys is about 45 minutes. Data is collected from participants at baseline (pre-intervention), 4 months post-accrual (immediate post-intervention), and 6 months post-accrual (Figure 2). Bowel function is assessed through the Memorial Sloan Kettering Cancer Center (MSKCC) Bowel Function Tool. This tool is designed to assess bowel function in RC, and includes 18 items with 3 subscales (frequency, dietary, urgency/soilage) and one total score. The responses are given on a 5-point Likert scale, apart from the frequency of bowel movements item. Construct validity 
was investigated, and psychometric properties have proved satisfactory, with Cronbach's alpha coefficient of $0.87 .34,35$

HRQOL is assessed using the City of Hope-Quality of Life-Colorectal Cancer (COHQOL-CRC). This tool contains categorical items used to identify demographic, disease, treatment, diet, work, and activity characteristics. It also contains a multi-item scale that assesses overall QOL and four QOL dimensions: physical, psychological, social, and spiritual well-being. Survey items are scaled from 0 to 10 with the higher number reflecting better HRQOL. The overall instrument remained reliable for ostomates (Cronbach’s alpha $=0.94)$ and non-ostomates $($ Cronbach’s alpha $=0.93) .{ }^{36}$ Separate ostomy and anastomosis versions are available.

The Low Anterior Resection Syndrome (LARS) Score is a 5-item survey with a corresponding value weighted according to the impact of bowel symptoms (low anterior resection syndrome) on QOL. ${ }^{37}$ Scores range from 0 to 42 points; scores are categorized into three groups: no LARS (0-20), minor LARS (21-29), and major LARS (30-42). The survey and scoring system have been validated in multi-national cohorts. ${ }^{5,38-41}$ This tool will be used for eligibility screening only.

Diet quality is assessed using the self-reported 24-hour recalls to estimate Healthy Eating Index (HEI-2010). The HEI-2010 scoring is based on a scale of 1 to 100 that measure adherence to the Dietary Guidelines for Americans, which aim to reduce diet-related chronic disease. A higher HEI-2010 score, is reflective of higher adherence to the guidelines. ${ }^{42}$ Such scoring system takes into consideration consumption of total fruit, whole fruits, total vegetables, total grains, dairy, total protein foods, total grains, empty calories, greens and beans, seafood, plant proteins, fatty acids, saturated fat and oils, and refined grains. For intakes consumed at the recommendation level or higher, a maximum score is received, where anything under the 
recommendation is scored proportionally. Scores of all individual foods are added together in a total score. $^{42}$

Finally, intervention acceptability will be determined using an investigator-developed Exit Interview Discussion Guide that will assess the following: 1) timing of the intervention; 2) length of sessions; 3) telephone-based approach; 4) usefulness of the session content; and 5) satisfaction with interventionists. In addition, we will also conduct interviews at the end of the study with health coaches to obtain feedback on intervention dose, overall participant engagement, and strategies to refine the intervention for future trials.

\section{Data Analysis}

Feasibility will be assessed through: 1) the ratio of eligible participants to those enrolled and those who declines participation; 2) reasons for non-participation; 3) number of scheduled study encounters completed; 3) attrition rate between pre- and post-intervention; 4) reasons for attrition/dropout; 5) level of intervention participation including the total number of sessions, number of sessions completed without break-offs, number of break-offs, length (minutes) of sessions; and 6) the ratio of all participants to those who completed $\geq 80 \%$ (8 out of 10 sessions) of the intervention. Acceptability will be assessed through qualitative data analysis using the conventional content analysis approach. ${ }^{43}$ This approach is used to describe a phenomenon where existing theory or research literature is limited. Data from the tape-recorded interviews will be transcribed. All data will be read repeatedly to achieve immersion and obtain a sense of the whole. Then, data will be read word by word to derive codes. Codes will then be sorted into themes based on links and relationship. Separate investigators will conduct a final validation review of the codes and themes to ensure consistency and clarity across all qualitative data. Data discordantly coded will be discussed for refinement and consensus purposes. 


\section{Discussion}

For rectal cancer survivors, persistent bowel dysfunction significantly impacts all aspects of HRQOL. To our knowledge, the AIMS intervention is among the first and few to address diet behavior changes for symptom management in rectal cancer survivors, an understudied cancer survivorship population. Despite the fact that bowel symptoms are commonly reported and modifiable with diet, the problem largely has been ignored. Recent published studies using electronic patient-reported symptoms and real-time symptom management have resulted in better health outcomes, fewer undesirable healthcare resource use (i.e. emergency department visits), and possibly extended survival. ${ }^{44,45}$ This suggests that interventions that address the symptom management needs of cancer survivors have great potential to improve health outcomes. Specifically, interventions to enhance self-efficacy and self-management of persistent symptoms are imperative in order to achieve the best possible outcomes for rectal cancer survivors. ${ }^{46}$ Our project is timely, innovative, and unique for several reasons. First, many challenges remain for cancer survivors, despite increasing awareness of survivorship issues after treatment. A major challenge is the lack of evidence-based guidelines and evidence-based interventions for quality post-treatment symptom management. The AIMS intervention addresses a unique long-term treatment effect of rectal cancer survivors, and can serve as a national model for comprehensive, quality survivorship care.

The AIMS intervention has great potential for improving bowel symptoms and HRQOL for rectal cancer survivors because of its grounding in the evidence-based behavior change and self-management theories. Further, our telephone approach in delivery of the intervention speaks to the potential for rapid implementation, dissemination, and scalability across different institutions, communities, and geographic locations. Telephone delivery offers many advantages, 
including greater access, affordability, and convenience for participants. From a scientific perspective, it allows for higher adherence to quality intervention fidelity through centralized intervention administration. Lastly, the telephone approach is recognized as a useful, important healthcare option for underserved populations. Those with access disparities, such as rural populations, elderly, inner-city, and ill patients, commonly have difficulty accessing specialty care. Our telephone approach offers the opportunity for trained health coaches to care for patients who are unable to access high-quality face-to-face care in an affordable, convenient manner.

In summary, there is an urgent need to advance the care of rectal cancer survivors to assure improved bowel function, diet quality and HRQOL after treatment. This pilot study will inform the refinement and design of future Phase II and Phase III trials of the AIMS Intervention. Overall, the study and design has a high probability of success because it is built on solid theoretical and practical understanding of the bowel issues facing rectal cancer survivors. 
Acknowledgements: Research reported in this article is supported by The Hope Foundation SEED Funds for SWOG Early Exploration and Development. The research reported in this article is also supported by the National Cancer Institute (NCI) of the National Institutes of Health (NIH) under award number P30CA33572 and P30CA023074. The statements presented in this article are solely the responsibility of the author(s) and do not necessarily represent the views of The Hope Foundation, the NCI, or the NIH. 


\section{References}

1. Miller KD, Siegel RL, Lin CC, et al. Cancer treatment and survivorship statistics, 2016. CA Cancer J Clin. Jul 2016;66(4):271-289.

2. Siegel RL, Miller KD, Jemal A. Cancer Statistics, 2017. CA Cancer J Clin. Jan 2017;67(1):730.

3. Herrinton LJ, Altschuler A, McMullen CK, et al. Conversations for providers caring for patients with rectal cancer: Comparison of long-term patient-centered outcomes for patients with low rectal cancer facing ostomy or sphincter-sparing surgery. CA Cancer J Clin. Mar 21 2016:n/a-n/a.

4. Sun V, Grant M, Wendel CS, et al. Dietary and Behavioral Adjustments to Manage Bowel Dysfunction After Surgery in Long-Term Colorectal Cancer Survivors. Annals of surgical oncology. Dec 2015;22(13):4317-4324.

5. Emmertsen KJ, Laurberg S, Rectal Cancer Function Study G. Impact of bowel dysfunction on quality of life after sphincter-preserving resection for rectal cancer. The British journal of surgery. Sep 2013;100(10):1377-1387.

6. Lynn PB, Renfro LA, Carrero XW, et al. Anorectal Function and Quality of Life in Patients With Early Stage Rectal Cancer Treated With Chemoradiation and Local Excision. Diseases of the colon and rectum. May 2017;60(5):459-468.

7. Kinoshita Y, Nokes KM, Kawamoto R, et al. Health-related quality of life in patients with lower rectal cancer after sphincter-saving surgery: a prospective 6-month follow-up study. European journal of cancer care. Jul 2017;26(4):e12417-n/a. 
8. Wiltink LM, Nout RA, van der Voort van Zyp JR, et al. Long-Term Health-Related Quality of Life in Patients With Rectal Cancer After Preoperative Short-Course and Long-Course (Chemo) Radiotherapy. Clinical colorectal cancer. Sep 2016;15(3):e93-99.

9. Wiltink LM, Marijnen CA, Meershoek-Klein Kranenbarg E, et al. A comprehensive longitudinal overview of health-related quality of life and symptoms after treatment for rectal cancer in the TME trial. Acta oncologica. 2016;55(4):502-508.

10. Steineck G, Schmidt H, Alevronta E, et al. Toward Restored Bowel Health in Rectal Cancer Survivors. Seminars in Radiation Oncology. 7// 2016;26(3):236-250.

11. Sun V, Wendel CS, Demark-Wahnefried W, et al. Diet Modifications to Manage Bowel Dysfunction Symptoms in Rectal Cancer Survivors. Paper presented at: 8th Biennial Cancer Survivorship Research Conference2016; Washington, DC.

12. Demark-Wahnefried W, Rogers LQ, Alfano CM, et al. Practical clinical interventions for diet, physical activity, and weight control in cancer survivors. CA: a cancer journal for clinicians. May 2015;65(3):167-189.

13. Van Loon K, Wigler D, Niedzwiecki D, et al. Comparison of Dietary and Lifestyle Habits Among Stage III and Metastatic Colorectal Cancer Patients: Findings from CALGB 89803 and CALGB 80405. Clinical colorectal cancer. 2013;12(2):95-102.

14. Meyerhardt JA, Niedzwiecki D, Hollis D, et al. Impact of body mass index and weight change after treatment on cancer recurrence and survival in patients with stage III colon cancer: Findings from cancer and leukemia group B 89803. Journal of Clinical Oncology. Sep 1 2008;26(25):4109-4115. 
15. Meyerhardt JA, Niedzwiecki D, Hollis D, et al. Association of dietary patterns with cancer recurrence and survival in patients with stage III colon cancer. JAMA. Aug 15 2007;298(7):754-764.

16. Cespedes Feliciano EM, Kroenke CH, Meyerhardt JA, et al. Metabolic Dysfunction, Obesity, and Survival Among Patients With Early-Stage Colorectal Cancer. J Clin Oncol. Sep 6 2016;34(30):3664-3671.

17. Hardcastle SJ, Maxwell-Smith C, Hagger MS, et al. Exploration of information and support needs in relation to health concerns, diet and physical activity in colorectal cancer survivors. Eur J Cancer Care (Engl). Mar 24 2017:e12679-n/a.

18. El-Shami K, Oeffinger KC, Erb NL, et al. American Cancer Society Colorectal Cancer Survivorship Care Guidelines. CA Cancer J Clin. Nov-Dec 2015;65(6):428-455.

19. Czajkowski SM, Powell LH, Adler N, et al. From ideas to efficacy: The ORBIT model for developing behavioral treatments for chronic diseases. Health psychology : official journal of the Division of Health Psychology, American Psychological Association. Oct 2015;34(10):971-982.

20. Thomson CA, Crane TE, Miller A, et al. A randomized trial of diet and physical activity in women treated for stage II-IV ovarian cancer: Rationale and design of the Lifestyle Intervention for Ovarian Cancer Enhanced Survival (LIVES): An NRG Oncology/Gynecologic Oncology Group (GOG-225) Study. Contemporary clinical trials. Jul 2016;49:181-189.

21. Coleman K, Austin BT, Brach C, et al. Evidence on the Chronic Care Model in the new millennium. Health affairs. Jan-Feb 2009;28(1):75-85. 
22. Wagner EH, Bennett SM, Austin BT, et al. Finding common ground: patient-centeredness and evidence-based chronic illness care. Journal of alternative and complementary medicine. 2005;11 Suppl 1:S7-15.

23. Wagner EH, Austin BT, Davis C, et al. Improving chronic illness care: translating evidence into action. Health affairs. Nov-Dec 2001;20(6):64-78.

24. Mosen DM, Schmittdiel J, Hibbard J, et al. Is patient activation associated with outcomes of care for adults with chronic conditions? The Journal of ambulatory care management. JanMar 2007;30(1):21-29.

25. Wagner EH. Chronic disease management: what will it take to improve care for chronic illness? Effective clinical practice : ECP. Aug-Sep 1998;1(1):2-4.

26. McCorkle R, Ercolano E, Lazenby M, et al. Self-management: Enabling and empowering patients living with cancer as a chronic illness. CA: a cancer journal for clinicians. Jan-Feb 2011;61(1):50-62.

27. Wagner EH, Austin BT, Von Korff M. Organizing care for patients with chronic illness. The Milbank quarterly. 1996;74(4):511-544.

28. Thomson C, Johnson C. Dietary Behaviors - Promoting Healthy Eating. In: Riekert K, Ockene J, Pbert L, eds. The Handbook of Health Behavior Change. 5th ed: Springer Publishing, 2017.

29. Moyers TB, Martin T, Christopher PJ, et al. Client language as a mediator of motivational interviewing efficacy: where is the evidence? Alcoholism, clinical and experimental research. Oct 2007;31(10 Suppl):40s-47s.

30. El-Shami K, Oeffinger KC, Erb NL, et al. American Cancer Society Colorectal Cancer Survivorship Care Guidelines. CA: a cancer journal for clinicians. Sep 82015. 
31. Meyerhardt JA, Mangu PB, Flynn PJ, et al. Follow-Up Care, Surveillance Protocol, and Secondary Prevention Measures for Survivors of Colorectal Cancer: American Society of Clinical Oncology Clinical Practice Guideline Endorsement. Journal of Clinical Oncology. December 10, 2013 2013;31(35):4465-4470.

32. Vitolins MZ, Rand CS, Rapp SR, et al. Measuring adherence to behavioral and medical interventions. Control Clin Trials. Oct 2000;21(5 Suppl):188S-194S.

33. Coday M, Boutin-Foster C, Sher TG, et al. Strategies for retaining study participants in behavioral intervention trials: Retention experiences of the nih behavior change consortium. Annals of Behavioral Medicine. April 01 2005;29(2):55-65.

34. Temple LK, Bacik J, Savatta SG, et al. The development of a validated instrument to evaluate bowel function after sphincter-preserving surgery for rectal cancer. Diseases of the colon and rectum. Jul 2005;48(7):1353-1365.

35. Wendel CS, Grant M, Herrinton L, et al. Reliability and validity of a survey to measure bowel function and quality of life in long-term rectal cancer survivors. Quality of life research : an international journal of quality of life aspects of treatment, care and rehabilitation. Dec 2014;23(10):2831-2840.

36. Grant M, Ferrell B, Dean G, et al. Revision and psychometric testing of the City of Hope Quality of Life-Ostomy Questionnaire. Quality of life research : an international journal of quality of life aspects of treatment, care and rehabilitation. Oct 2004;13(8):1445-1457.

37. Emmertsen KJ, Laurberg S. Low anterior resection syndrome score: development and validation of a symptom-based scoring system for bowel dysfunction after low anterior resection for rectal cancer. Annals of surgery. May 2012;255(5):922-928. 
38. Juul T, Battersby NJ, Christensen P, et al. Validation of the English translation of the low anterior resection syndrome score. Colorectal Dis. Oct 2015;17(10):908-916.

39. Chen TY, Emmertsen KJ, Laurberg S. What Are the Best Questionnaires To Capture Anorectal Function After Surgery in Rectal Cancer? Current colorectal cancer reports. 2015;11:37-43.

40. Juul T, Ahlberg M, Biondo S, et al. International validation of the low anterior resection syndrome score. Ann Surg. Apr 2014;259(4):728-734.

41. Bregendahl S, Emmertsen KJ, Lous J, et al. Bowel dysfunction after low anterior resection with and without neoadjuvant therapy for rectal cancer: a population-based cross-sectional study. Colorectal Dis. Sep 2013;15(9):1130-1139.

42. Guenther PM, Casavale KO, Reedy J, et al. Update of the Healthy Eating Index: HEI-2010. Journal of the Academy of Nutrition and Dietetics. Apr 2013;113(4):569-580.

43. Hsieh HF, Shannon SE. Three approaches to qualitative content analysis. Qualitative health research. Nov 2005;15(9):1277-1288.

44. Basch E, Deal AM, Kris MG, et al. Symptom Monitoring With Patient-Reported Outcomes During Routine Cancer Treatment: A Randomized Controlled Trial. J Clin Oncol. Feb 20 2016;34(6):557-565.

45. Basch E, Snyder C, McNiff K, et al. Patient-reported outcome performance measures in oncology. Journal of oncology practice / American Society of Clinical Oncology. May 2014;10(3):209-211.

46. Dayal S, Battersby N, Cecil T. Evolution of Surgical Treatment for Rectal Cancer: a Review. Journal of gastrointestinal surgery : official journal of the Society for Surgery of the Alimentary Tract. Jul 2017;21(7):1166-1173. 